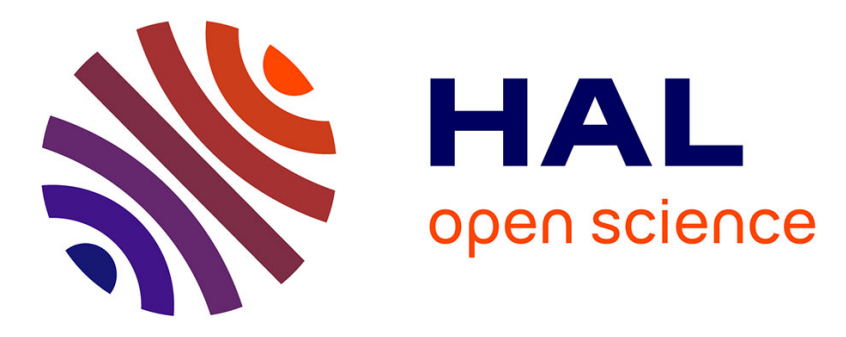

\title{
Tight approximation for scheduling parallel jobs on identical clusters
}

\author{
Marin Bougeret, Pierre-Francois Dutot, Klaus Jansen, Christina Robenek, \\ Denis Trystram
}

\section{- To cite this version:}

Marin Bougeret, Pierre-Francois Dutot, Klaus Jansen, Christina Robenek, Denis Trystram. Tight approximation for scheduling parallel jobs on identical clusters. IPDPSW: International Parallel and Distributed Processing Symposim, May 2012, Shangai, China. pp.878-885, 10.1109/IPDPSW.2012.108 . hal-00738499

\section{HAL Id: hal-00738499 \\ https://hal.science/hal-00738499}

Submitted on 4 Oct 2012

HAL is a multi-disciplinary open access archive for the deposit and dissemination of scientific research documents, whether they are published or not. The documents may come from teaching and research institutions in France or abroad, or from public or private research centers.
L'archive ouverte pluridisciplinaire HAL, est destinée au dépôt et à la diffusion de documents scientifiques de niveau recherche, publiés ou non, émanant des établissements d'enseignement et de recherche français ou étrangers, des laboratoires publics ou privés. 


\title{
Tight approximation for scheduling parallel jobs on identical clusters
}

\author{
Marin Bougeret, Pierre-François Dutot’, Klaus Jansen; \\ Christina Robenek, ${ }^{\ddagger}$ Denis Trystram ${ }^{\dagger \top}$
}

\begin{abstract}
We consider the Multiple Cluster Scheduling Problem $(M C S P)$, where the objective is to schedule $n$ parallel rigid jobs on $N$ identical clusters, minimizing the maximum completion time (makespan). MCSP is 2-inapproximable (unless $\mathcal{P}=\mathcal{N} \mathcal{P}$ ), and several approximation algorithms have already been proposed. However, ratio 2 has only been reached by algorithms that use extremely costly and complex subroutines as "black boxes" which are polynomial and yet impractical due to prohibitive constants.

Our objective within this work is to determine a reasonable restriction of MCSP where the inapproximability lower bound could be tightened in almost linear time. Thus, we consider a restriction of MCSP where jobs do not require strictly more than half of the processors of a cluster, and we provide a 2-approximation running in $O\left(\log \left(n h_{\max }\right) n(N+\log (n))\right)$, where $h_{\max }$ is the processing time of the longest job. This approximation is the best possible, as this restriction (and even simpler ones) remains 2-inapproximable.
\end{abstract}

\section{Introduction}

\subsection{Problem statement}

In new parallel computing platforms, several clusters share their computing resources in order to distribute the workload. Each cluster is composed of a set of identical processors connected by a local interconnection network. Jobs are submitted in successive packets called batches. The objective is to minimize the time when all the jobs of a batch are completed, to start the following batch as soon as possible. Many such computational systems are available all over the world, and the efficient management of the resources is a crucial problem. Let us start by defining formally the Multiple Cluster Scheduling Problem (MCSP).

\footnotetext{
* LIRMM, Montpellier

† Department of Computer Science, University Kiel, Germany

$\dagger$ Grenoble University

ฯ Institut Universitaire de France
}

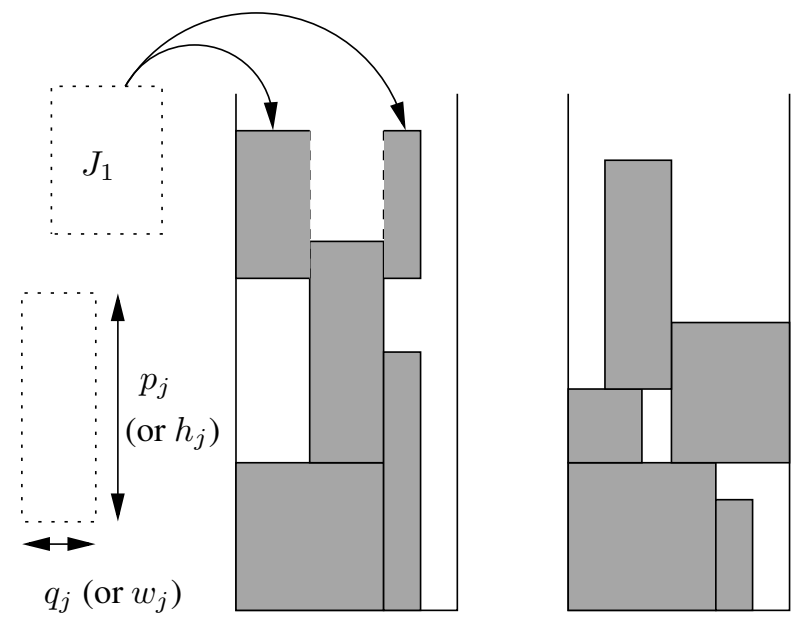

Figure 1. Example for 9 jobs and 2 clusters of a feasible solution for $M C S P$ and not feasible for $M S P P$.

Definition 1 (MCSP) We are given $n$ parallel rigid jobs $J_{j}, 1 \leq j \leq n$, and $N$ clusters. A job $J_{j}$ requires $q_{j}$ processors during $p_{j}$ units of time, and each cluster owns $m$ identical processors. The objective is to schedule all the jobs in the clusters, minimizing the maximum completion time (makespan) under the following constraints:

1. the $q_{j}$ processors allocated to job $J_{j}$ must belong to the same cluster (as proposed in [5])

2. at any time, the total number of processors used in any cluster must be lower or equal to $m$

$M C S P$ is closely related to the Multiple Strip Packing Problem $(M S P P)$ where the objective is to pack $n$ rectangles in $N$ strips, minimizing the maximum reached height. The only difference between these two problems is the "contiguous" constraint. Indeed, in MSPP rectangles must be allocated contiguously, which in terms of job scheduling amounts to force jobs to use consecutive indexes of processors (see Figure 1).

Of course, the results for $M C S P$ generally do not apply to $M S P P$, as algorithms may schedule jobs in a non con- 
tiguous way. The converse is also not clear, as ratio of approximation algorithms for $M S P P$ may not be preserved when considering $M C S P$ (optimal value of an $M C S P$ instance may be strictly better than the corresponding one for $M S P P)$. However, as we can notice in Figure 2, many results for $M S P P$ directly apply to $M C S P$, as the proposed algorithms build contiguous schedules that are compared to non-contiguous optimal solutions.

We refer to the packing context in this paper, so that the solutions can be described using the classical vocabulary of packing problems. Thus, the problem treated in this paper $(M C S P)$ consists in packing $n$ rectangles in $N$ strips of width 1 , minimizing the maximum reached height, and without contiguous constraints ${ }^{1}$.

\subsection{Related Work}

As shown in [11] using a gap reduction from 2 partition, $M C S P$ (and $M S P P$ ) are 2-inapproximable in polynomial time unless $\mathcal{P}=\mathcal{N} \mathcal{P}$, even for $N=2$. The main positive results for MCSP are summarized below, in Figure 2.

\begin{tabular}{|l|c|l|c|}
\hline Problem & Ratio & Remarks & Source \\
\hline \hline $\begin{array}{l}M C S P, \\
M S P P\end{array}$ & $2 \rho$ & $\begin{array}{l}\text { Need solving } P \| C_{\max } \\
\text { with a ratio } \rho\end{array}$ & {$[10]$} \\
\hline$M C S P$ & $5 / 2$ & Fast algorithm & {$[3]$} \\
\hline $\begin{array}{l}M C S P, \\
M S P P\end{array}$ & 2 & $\begin{array}{l}\text { Need applying the PTAS } \\
\text { of [1] for a constant } \approx \\
\left.10^{4}\right) \text { number of clusters }\end{array}$ & {$[2]$} \\
\hline $\begin{array}{l}M C S P, \\
M S P P\end{array}$ & AFPTAS & $\begin{array}{l}\text { Additive constant in } \\
\mathcal{O}\left(\frac{1}{\epsilon^{2}}\right), \text { and in } \mathcal{O}(1) \text { for } \\
\text { large values of } N\end{array}$ & {$[2]$} \\
\hline$M C S P$ & 3 & $\begin{array}{l}\text { Fast }(\text { and decentralized }) \\
\text { algorithm handling } \\
\text { clusters of different size }\end{array}$ & {$[9]$} \\
\hline$M C S P$ & 2 & $\begin{array}{l}\text { Requires } \max _{j} w_{j} \leq \frac{1}{2} \\
\text { Fast algorithm }\end{array}$ & this paper \\
\hline
\end{tabular}

Figure 2. Main results

We distinguish the 3-approximation [9] and the $\frac{5}{2}$ approximation [3] that have a low computational complexity (these algorithms are usable on real size instances) from the 2-approximation [2] and the $2+\epsilon$-approximation [10].

At a first sight, the best results seem to be the 2approximation in [2] and the $2+\epsilon$-approximation in [10]. However, the 2-approximation requires using a high running time algorithm when the number of clusters is lower than a huge constant $N_{0}$. Thus, any exponential dependency in $N_{0}$ is hidden, and the value of this constant $\left(N_{0} \approx 10^{4}\right)$

\footnotetext{
${ }^{1}$ Therefore, when trying to pack a rectangle of width $w_{j}$ at level $l$ of a strip, we only need to check if the occupation at level $l$ is lower than $1-w_{j}$.
}

makes this algorithm practically impossible to use. Moreover, the $2+\epsilon$-approximation requires solving the famous $P \| C_{\max }$ problem (which is makespan minimization when scheduling sequential jobs on identical machines) with a ratio $1+\frac{\epsilon}{2}$. Thus, to give a rough idea, applying this technique with $\epsilon=\frac{1}{3}$ would lead to a $\Omega\left(n^{36}\right)$ algorithm, using the PTAS of [6]. ${ }^{2}$

\subsection{Motivations and contributions}

As explained above, the two previous results of $[2,10]$ require both extremely high running time, and do not provide any insight on $M C S P$ because of these black boxes subroutines. Thus, we followed in [3] another approach by looking for fast and direct algorithms for $M C S P$. Our previous $\frac{5}{2}$-approximation in [3] is based on the discarding technique presented in Section 2.2. What we call discarding technique is a classical framework in scheduling problems. The idea is to define properly a set of negligeable items (items are rectangles here), and to prove that it is possible to add these items only at the end of the algorithm without degrading the approximation ratio. Thus, the effort can be focused on the set $I^{\prime}$ of remaining large items, that are generally more structured.

The $\frac{5}{2}$-approximation was obtained through a basic application of this technique (i.e. with a set $I^{\prime}$ containing only really huge rectangles, in this case rectangles whose width is larger than $\frac{1}{2}$ ). As we believe that the discarding technique of Section 2.2 is well suited for $M C S P$, we apply it again using a more challenging set $I^{\prime}$. A natural direction would be to improve the $\frac{5}{2}$ ratio for $M C S P$ by targeting a fixed ratio $\rho<\frac{5}{2}$. Typically, one could target $\rho=\frac{7}{3}$ by defining the small jobs as those whose length is lower than $\frac{1}{3}$ (instead of $\frac{1}{2}$ ). However, as the relative performance improvement is getting smaller, and the difficulty of these "ratio tailored" proofs is likely to increase rapidly, we consider here a different approach.

Our objective is to find a reasonable restriction of $M C S P$ where the inapproximability lower bound could be tightened in almost linear time. In this spirit, we study a restriction of $M C S P$ where all rectangles have a width lower than $\frac{1}{2}$, meaning that jobs submitted to the clusters do not require strictly more than half of the processors. We provide for this problem a very fast 2-approximation running in $O\left(\log \left(n h_{\max }\right) n(N+\log (n))\right)$, where $h_{\max }$ is the maximum height of any rectangle. It turns out that this result is the best possible approximation, as this restriction of MCSP (and even simpler ones, where the width of rectangles is lower than $\frac{1}{c}, c \in \mathbb{N}, c \geq 2$ ) remains 2inapproximable unless $\mathcal{P}=\mathcal{N} \mathcal{P}$.

\footnotetext{
${ }^{2}$ Even if some recent advances in the PTAS design for $P \| C_{\max }$ allowed to decrease the asymptotic depedencies in $\frac{1}{\epsilon}\left(\right.$ like $\left.2^{\mathcal{O}\left(\frac{1}{\epsilon^{2}} \log ^{3}\left(\frac{1}{\epsilon}\right)\right)}\right)$ in [8], the running time of these algorithms remain high due to constants.
} 


\section{General principles}

In this section, we generalize the framework used in the $\frac{5}{2}$-approximation of [3]. This framework will be applied in Section 3 to get the 2-approximation.

\subsection{Preliminaries}

Recall that our objective is to (non contiguously) pack a set $I$ of $n$ rectangles $r_{j}$ into $N$ strips of width 1 . Rectangle $r_{j}$ has a height $h_{j}$ and a width $w_{j}$. We denote by $s\left(r_{j}\right)=$ $w_{j} h_{j}$ the surface of $r_{j}$. These notations are extended to $W(X), H(X)$ and $S(X)$ (where $X$ is a set of rectangles), which denote the sum of the widths (resp. heights, surfaces) of rectangles in $X$.

A layer is a set of rectangles packed one on top of the other in the same strip (as depicted Figure 3). The height of layer Lay is H(Lay), the sum of the height of all the rectangles in Lay. A shelf is a set of rectangles that are packed in the same strip, such as the bottom level of all the rectangles is the same. Even if it is not relevant for the noncontiguous case, we consider for the sake of simplicity that in a shelf, the right side of any rectangle (except the right most one) is adjacent to the left side of the next rectangle in the shelf. Given a shelf $s h$ ( $s h$ denotes the set of rectangles in the shelf), the value $W(s h)$ is called the width of $s h$. Packing a shelf at level $l$ means that all the rectangles of the shelf have their bottom at level $l$. A bin is a rectangular area that can be seen as a reserved space in a particular strip for packing rectangles. As the width of a bin is always 1, we define a bin by giving its height $h_{b}$, its bottom level $l_{b}$ and the index $i_{b}$ of the strip it belongs to. Packing a shelf $s h$ in a bin $b$ means that $s h$ is packed in strip $S_{i_{b}}$ at level $l_{b}$. Moreover we always guarantee that the height of any rectangle of $s h$ is lower than $h_{b}$.

The utilization $u_{i}^{\pi}(l)$ of a packing $\pi$ in strip $S_{i}$ at level $l$ (sometimes simply denoted by $u(l)$ or $u_{i}(l)$ ) is the sum of the width of all the rectangles packed in $S_{i}$ that cut the horizontal line-level $l$ (see Figure 3). Of course we have $0 \leq u_{i}^{\pi}(l) \leq 1$ for any $l$ and $i$.

Let us now describe three useful basic procedures. The CreateLayer $(X, h)$ procedure creates a layer Lay (using rectangles of $X$ ) of height at most $h$, using a Best Fit (according to the height) policy (BFH). Thus, CreateLayer $(X, h)$ adds at each step the highest rectangle that fits. Of course, the layer produced by the procedure is such that $H(L a y) \leq h$. Moreover, notice that we will always pack the layers in the strips with the narrowest rectangles on the top. CreateShelf $(X, w)$ creates a shelf $s h$ (using rectangles of $X$ ) of width at most $w$, using the Best Fit (according to the width) policy (BFW). Thus, CreateShelf $(X, w)$ adds at each step the widest rectangle that fits. Of course, the shelf produced by the procedure is

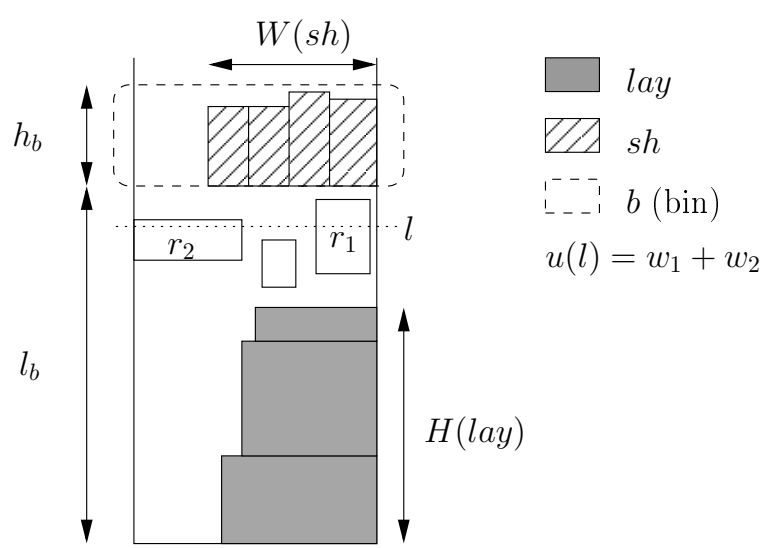

Figure 3. Example of a layer, a shelf, a bin and of the utilization function. $s h$ is packed in $b$.

such that $W(s h) \leq w$. Throughout the paper, we consider that the sets of jobs used as parameters in the algorithms are modified after the calls.

Let us now state a standard lemma about the efficiency of the "best fit" policies.

Lemma 2 Let $S h$ denote the shelf created by CreateShelf $(X, w)$. If the $k$ widest rectangles of $X$ are added to $S h$, then $W(S h)>\frac{k}{k+1} w$.

Proof Let $x$ be the cardinality of $X$. Let us assume that $w_{i} \geq w_{i+1}$ for $1 \leq i<x$. Let $i_{0} \geq k+1$ be the first index such that $r_{i_{0}}$ is not in $S h$. Let $a=\sum_{i=1}^{i_{0}-1} w_{i}$. We have $W(S h) \geq a \geq\left(i_{0}-1\right) w_{i_{0}}>\left(i_{0}-1\right)(w-a)$ leading to $a>\frac{i_{0}-1}{i_{0}} w \geq \frac{k}{k+1} w$.

\subsection{Discarding technique}

\subsubsection{How to pack all rectangles in three steps}

Discarding techniques are common for solving packing and scheduling problem. As mentioned before, the idea is to define properly a set of small items (rectangles here), and to prove that adding these small items only at the end of the algorithm will not degrade the approximation ratio. Thus, the effort can be focused on the remaining large items. In this section we present an adaptation of this general technique to the context of non-contiguous multiple strip packing. Thus, we will define a set of big rectangles $I^{\prime} \subset I$, and the larger the set $I^{\prime}$, the better the approximation ratio (as the remaining small rectangles become really negligible).

In order to partition rectangles according to their height, we use the well-known dual approximation technique [7]. 
We denote by $v$ the guess of the optimal value. Given an instance $I$, let $L_{W D}=\left\{w_{j}>\alpha\right\}$ be the set of wide rectangles, $L_{H}=\left\{h_{j}>\beta v\right\}$ be the set of high rectangles, and $I^{\prime}=L_{W D} \cup L_{H}$ be the set of the big rectangles, with $0<\alpha<1$ and $0<\beta<1$. Let $r(\alpha, \beta)=\frac{1}{1-\alpha}+\beta$ be the approximation ratio we target (the origin of this formula will be explained in Section 2.2.2). We also need the following definition.

Definition 3 A packing is x-compact if and only if for every strip $S_{i}$ there exists a level $l_{i}$ such that for all $l \leq l_{i}, u_{i}(l)>$ $x$ and $u_{i}$ restricted to $l>l_{i}$ is non-increasing.

Figure 4 provides an example showing a $(1-\alpha)$ compact packing, and why step c) is simple. Indeed, adding as soon as possible a small rectangle $r_{j}\left(h_{j} \leq \beta v\right.$ and $\left.w_{j} \leq \alpha\right)$ to a $(1-\alpha)$ compact packing cannot exceed $v\left(\frac{1}{1-\alpha}+\beta\right)$.

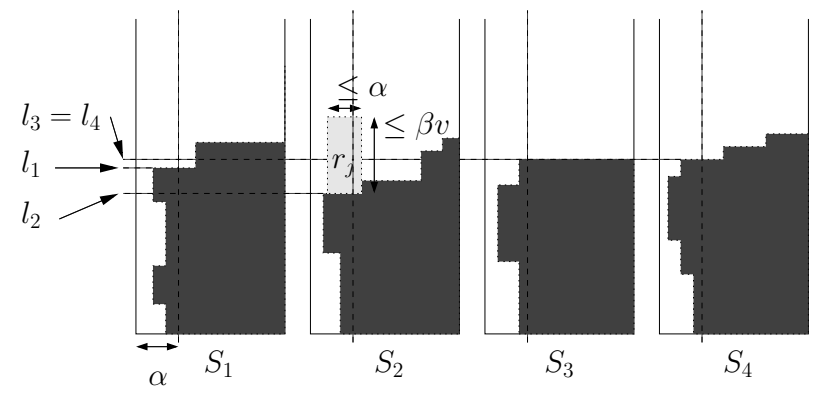

Figure 4. $(1-\alpha)$ compact packing

Let us now describe the three main steps of our approach. Notice that what we call a preallocation is a normal packing (i.e. that defines the bottom level of each rectangle, which is sufficient) that is based on simple structures like shelves and layers. We will prove that to get a $r(\alpha, \beta)=\left(\frac{1}{1-\alpha}+\beta\right)$ ratio, it is sufficient to:

a) construct a preallocation $\pi_{0}$ of $I^{\prime}$ that fits in $r(\alpha, \beta) v$, and such that rectangles of $L_{W D} \subset I^{\prime}$ are already packed in a $(1-\alpha)$-compact way

b) turn $\pi_{0}$ into a $(1-\alpha)$-compact packing $\pi_{1}$ by repacking rectangles of $I^{\prime} \backslash L_{W D}$ using the list algorithm $L S_{\pi_{0}}$ of Lemma 4

c) add the small remaining rectangles $\left(I \backslash I^{\prime}\right)$ using algorithm $L S$ (see Lemma 5)

Step a) is the most difficult one. Thus, Section 3 is entirely devoted to the construction of $\pi_{0}$ (targeting $\alpha=\frac{1}{3}$ and $\beta=\frac{1}{2}$ ). Of course, building the preallocation becomes harder when $\alpha$ and $\beta$ are small, as the number of rectangles of $I^{\prime}$ increases and $r(\alpha, \beta)$ decreases. Roughly speaking, the simple shapes of rectangles of $I^{\prime}$ allows us to construct $\pi_{0}$ with a simple structure. We will denote by $\pi_{0}^{i}$ the set of rectangles packed by $\pi_{0}$ in $S_{i}$.

\subsubsection{Proving steps b) and c)}

We now prove that applying steps b) and c) leads to a $r(\alpha, \beta)$ ratio. In this section, we suppose that we are given a guess $v$, and a packing $\pi_{0}$ (called the preallocation) of $I^{\prime}=L_{W D} \cup L_{H}$ that fits in $r(\alpha, \beta) v$, and such that rectangles of $L_{W D} \subset I^{\prime}$ are already packed in a $(1-\alpha)$-compact way. We consider step b): how to turn $\pi_{0}$ into a $(1-\alpha)$ compact packing.

Lemma 4 (Step b)) Let $\pi_{0}$ be the preallocation of $I^{\prime}$ constructed in Step a). Let $\widehat{\pi_{1}}=\pi_{0} \cap L_{W D}$ denote $\pi_{0}$ when keeping only rectangles of $L_{W D}$. Recall that $\widehat{\pi_{1}}$ is already $a(1-\alpha)$-compact packing of rectangles of $L_{W D}$.

Then, we can complete $\widehat{\pi_{1}}$ into a $(1-\alpha)$-compact packing $\pi_{1}$ of $I^{\prime}$, such that the height of $\pi_{1}$ is lower or equal to the height of $\pi_{0}$.

Proof Let us define the $L S_{\pi_{0}}$ algorithm that adds rectangles of $I^{\prime} \backslash L_{W D}$. Let us consider a single strip $S_{i}$. Let $\pi_{0}^{i}$ denote $\pi_{0}$ restricted to $S_{i}$, and $\widehat{\pi_{1}^{i}}$ denote $\widehat{\pi_{1}}$ restricted to $S_{i}$. Let $X=\left\{r_{1}, \ldots, r_{p}\right\}$ be the set of preallocated rectangles of $I^{\prime} \backslash L_{W D}$ that we have to add to $S_{i}$. We assume that $\operatorname{lvl}(j) \leq l v l(j+1)$, where $\operatorname{lvl}(j)$ is the bottom level of $r_{j}$ in $\pi_{0}$.

For our considered strip $S_{i}$, the $L S_{\pi_{0}}$ algorithm executes $\operatorname{AddAsap}\left(r_{j}, \widehat{\pi_{1}^{i}}\right)$, for $1 \leq j \leq p$, where $\operatorname{Add} A \operatorname{sap}\left(r, \widehat{\pi_{1}^{i}}\right)$ adds rectangle $r$ to $\widehat{\pi_{1}}$ (in $S_{i}$ ) at the smallest possible level. Notice first that adding with AddAsap a rectangle $r_{j}$ with $w_{j} \leq \alpha$ to a $(1-\alpha)$-compact packing creates another $(1-$ $\alpha)$-compact packing. Thus it is clear that $\pi_{1}$ is $(1-\alpha)$ compact.

For any $1 \leq j \leq p$, let $\left.\widehat{\pi_{1}^{i}}, j\right)$ denote the packing in $S_{i}$ just before adding $r_{j}$ with AddAsap, and let $\left(\pi_{0}^{i}, j\right)$ denote the packing $\pi_{0}^{i} \cap\left(L_{W D} \cup\left\{r_{1}, \ldots, r_{j-1}\right\}\right)$. Let us prove by induction on $j \in\{1, \ldots, p\}$ that $u^{\left(\widehat{\pi_{1}^{i}}, j\right)}(l) \leq u^{\left(\pi_{0}^{i}, j\right)}(l)$, for any $l \geq l v l(j)$. The definition of $\widehat{\pi_{1}}$ implies the property for $j=1$ (packings are identical). Let us suppose that the property holds for $j$, and prove it for $j+1$. Let $l \geq \operatorname{lvl}(j+1)$. The induction property for rank $j$ implies that $r_{j}$ is added by AddAsap at a level lower or equal to $\operatorname{lvl}(j)$. Thus, if $r_{j}$ intersects $l$ in $\left.\widehat{\left(\pi_{1}^{i}\right.}, j+1\right)$, then it also occurs in $\left(\pi_{0}^{i}, j+1\right)$. Thus in this case we have

$$
\begin{aligned}
u^{\left.\widehat{\left(\pi_{1}^{i}\right.}, j+1\right)}(l) & =u^{\left(\widehat{\pi_{1}^{i}}, j\right)}(l)+w_{j} \\
& \leq u^{\left(\pi_{0}^{i}, j\right)}(l)+w_{j} \\
& =u^{\left(\pi_{0}^{i}, j+1\right)}(l)
\end{aligned}
$$

If $r_{j}$ does not intersect $l$ in $\left.\widehat{\left(\pi_{1}^{i}\right.}, j\right)$, then clearly $u^{\left(\widehat{\pi_{1}^{i}}, j+1\right)}(l)=u^{\left(\widehat{\pi_{1}^{i}}, j\right)}(l) \leq u^{\left(\pi_{0}^{i}, j\right)}(l) \leq u^{\left(\pi_{0}^{i}, j+1\right)}(l)$

Thus we proved that for any $1 \leq j \leq p$ we have $u^{\left(\widehat{\pi_{1}^{i}}, j\right)}(l) \leq u^{\left(\pi_{0}^{i}, j\right)}(l)$ for any $l \geq \operatorname{lvl}(j)$, implying that 
every $r_{j}$ is added by AddAsap at a level lower or equal to $\operatorname{lvl}(j)$. Thus, the height of $\pi_{1}$ is lower or equal to the height of $\pi_{0}$

We now prove in Lemma 5 that after adding rectangles in step c), the height of the packing does not exceed $r(\alpha, \beta) v=\left(\frac{1}{1-\alpha}+\beta\right) v$. This explains why the height of the pre-allocation should also be bounded by $r(\alpha, \beta) v$.

Lemma 5 (Step c)) Let $\pi_{1}$ be a $(1-\alpha)$-compact packing of $I^{\prime}$. Adding to $\pi_{1}$ rectangles of $I \backslash I^{\prime}$ with a List Scheduling algorithm ( $L S$ ) leads to a packing $\pi$ having height lower than $\max \left(\right.$ height $\left.\left(\pi_{1}\right), v\left(\frac{1}{1-\alpha}+\beta\right)\right)$.

Proof The $L S$ algorithm scans all the strips from level 0, and at any level adds any rectangle of $I \backslash I^{\prime}$ that fits. Notice that the final packing $\pi$ is $(1-\alpha)$-compact, since we add rectangles $r_{j}$ with $w_{j} \leq \alpha$ to an $(1-\alpha)$-compact packing.

Let us assume that the height of $\pi$ is due to a rectangle $r_{j} \in I \backslash I^{\prime}$ that starts at level $s$. This implies that when packing $r_{j}$ we had $l_{i} \geq s$ for any strip $i$ (with $l_{i}$ defined as in Definition 3). According to this definition we have $u_{i}(l)>1-\alpha$ for any $l \leq l_{i}$. Thus, we have $S(I)>\sum_{i=1}^{N} l_{i}(1-\alpha) \geq N(1-\alpha) s$, implying that $s<v \frac{1}{1-\alpha}$, and thus that of height of $\pi$ is lower or equal to $s+\max _{j \in I \backslash I^{\prime}} h_{j} \leq v\left(\frac{1}{1-\alpha}+\beta\right)$.

Thus, we now apply this framework with $\alpha=\frac{1}{3}$ and $\beta=\frac{1}{2}$ to get a 2 -approximation.

\section{2-approximation}

\subsection{Hardness}

As explained before, the $2+\epsilon$-approximation in [10] and the 2-approximation we recently proposed in [2] are rather complexity results than practical algorithms. We aim at constructing a low cost algorithm that could be used in a practical context. Thus, we are considering a restriction of $M C S P$ where the inapproximability bound could be tightened with a fast algorithm, and we consider that all the rectangles have a width lower or equal to $1 / 2$.

Theorem 6 The MCSP where every rectangle has a width lower (or equal) to $\frac{1}{2}$ has no polynomial algorithm with a ratio strictly better than 2 , unless $P=N P$.

Proof As in [11] for the general version, we construct a gap reduction from 2-partition. Let $\left\{x_{1}, \ldots, x_{n}\right\} \subset \mathbb{N}^{n}$ and $a$ such that $\sum_{i=1}^{n}=2 a$. Without loss of generality, let us assume that for any $i, x_{i}<a$. In order to only have items with size at least two, we define $x_{i}^{\prime}=2 x_{i}$ for any $i$, and $a^{\prime}=2 a$. We construct the following instance $I_{M S P}$ : $N=2$ strips, each of size $2 a^{\prime}-1$. The set of rectangles is $\left\{r_{1}, \ldots, r_{n}, r_{n+1}, r_{n+2}\right\}$, with $w_{i}=x_{i}^{\prime}$ for $1 \leq i \leq n$, $w_{n+1}=w_{n+2}=a^{\prime}-1$, and $h_{i}=1$ for $1 \leq i \leq n+2$. We have $w_{i} \leq \frac{2 a^{\prime}-1}{2}$ for any $i$, as all the $x_{i}$ are strictly lower than $a$. Notice than any solution of $I_{M S P}$ that packs $r_{n+1}$ and $r_{n+2}$ is the same strip has a height of at least 2 , as the available width of size 1 in that strip cannot be used by any rectangle.

Obviously, if there is a 2-partition, then $O p t\left(I_{M S P}\right)=$ 1. Otherwise, as $r_{n+1}$ and $r_{n+2}$ cannot be packed together, we have $O p t\left(I_{M S P}\right)=2$

The previous proof can easily be adapted for any non-trivial restriction on the size of the widest rectangle. Therefore, the fast 2-approximation presented in this section is the best possible result, even for more restricted versions of the MCSP.

\subsection{Decomposition}

We follow the ideas presented in Section 2, and thus we re-use the notion of layer, shelf, bin, and the procedures named CreateLayer and CreateShelf.

Again, we use the dual approximation technique [7], and we denote by $v$ the guess of the optimal value. Conforming to the dual approximation technique, we will prove that either we pack $I$ with a resulting height lower than $2 v$, or $v<O p t$. Then, we will perform a binary search on $v$ to turn the dual approximation algorithm into a classical approximation algorithm. Notice that for the sake of simplicity we did not add the "reject" instructions in the algorithm. Thus we consider in all the proof that $v \geq O p t$, and it is implicit that if one of the claimed properties is wrong during the execution, the considered $v$ should be rejected.

Recall that all rectangles have $w_{j} \leq \frac{1}{2}$. Let us define the following sets:

- let $L_{W D}=\left\{r_{j} \mid w_{j}>1 / 3\right\}$ be the set of wide rectangles

- let $L_{X H}=\left\{r_{j} \mid h_{j}>2 v / 3\right\}$ be the set of very high rectangles

- let $L_{H}=\left\{\left.r_{j}\right|^{2 v / 3} \geq h_{j}>v / 2\right\}$ be the set of high rectangles

- let $L_{B}=\left(L_{X H} \cup L_{H}\right) \cap L_{W D}$ be the set of huge rectangles, and $b=\operatorname{Card}\left(L_{B}\right)$.

- let $I^{\prime}=L_{W D} \cup L_{X H} \cup L_{H}$

Notice that we only consider the values $v$ such that $W\left(L_{X H} \cup L_{H}\right) \leq N$ and $H\left(L_{W D}\right) \leq 2 N v$.

As expected, the set $I^{\prime}$ corresponds in our framework to the set of big rectangles for $\alpha=\frac{1}{3}$ and $\beta=\frac{1}{2}$. The 
construction of the preallocation $\pi_{0}$ of $I^{\prime}$ is presented from Section 3.3 to 3.5 . The final steps to turn $\pi_{0}$ into a $\frac{2}{3}$ compact packing $\pi_{1}$ and to turn $\pi_{1}$ into the final packing $\pi$ are quickly described in Section 3.6, as they follow the steps presented in Section 2.2.

We now provide a two phases algorithm that builds the preallocation $\pi_{0}$ of the rectangles of $I^{\prime}$. Phase 1 (Section 3.3) preallocates rectangles of $L_{W D}$, and phase 2 (Section 3.5) preallocates rectangles of $L_{H} \cup L_{X H}$.

\subsection{Phase 1}

Phase 1 packs the rectangles of $L_{W D}$ by calling for each strip (until $L_{W D}$ is empty) two times CreateLayer $\left(L_{W D}, 2 v\right)$. Let us denote by $\operatorname{Lay}_{2 i-1}$ and $L a y_{2 i}$ the layers created in strip $S_{i}$. Let us say that $L a y_{2 i-1}$ is packed left justified, and $L a y_{2 i}$ is packed right justified. Moreover, each layer is repacked in non increasing order of the widths, such that the narrowest rectangles are packed on the top.

Let $N_{1}$ denote the number of strips used in phase 1 , and let $i_{1}$ denote the index of the last created layer ( $L a y_{i_{1}}$ is of course in $S_{N_{1}}$ ). Let $L_{H}^{1}$ and $L_{X H}^{1}$ denote the set of remaining rectangles after phase 1 of $L_{H}$ and $L_{X H}$, respectively. Thus, for the moment we have $\pi_{0}^{i}=L a y_{2 i} \cup L a y_{2 i-1}$ for all $i \leq N_{1}$.

Lemma 7 If $\exists i_{0}<i_{1}$ such that $H\left(\operatorname{Lay}_{i_{0}}\right) \leq \frac{3 v}{2}$ then it is straightforward to preallocate $I^{\prime}$.

Proof Let $i_{0}<i_{1}$ such that $H\left(L a y_{i_{0}}\right) \leq \frac{3 v}{2}$. This implies that we ran out of rectangles of $L_{W D} \backslash\left(L_{H} \cup L_{X H}\right)$ while creating layer $i_{0}$. Thus, because of the $B F H$ order there are at least two rectangles of $L_{B}$ in every layer $L a y_{i}$, for $1 \leq i<i_{1}$, implying that the width of high and very high rectangles packed in each of these layers is strictly larger than $2 / 3$. Thus, $W\left(\pi_{0}^{i} \cap\left(L_{H} \cup L_{X H}\right)\right)>4 / 3>1$ for $1 \leq i<N_{1}$. Thus, the total width of remaining high and very high rectangles is lower than $N-\left(N_{1}-1\right)$.

Let us prove that we can pack all the remaining rectangles of $I^{\prime}$ (which are included in $\left(L_{H} \cup L_{X H}\right)$ ) in the remaining strips. For each $i \in\left[\left|N_{1}+1, N\right|\right]$ we create two shelves in $S_{i}$ (one at level 0 and one at level $v$ ). If there are still some unpacked rectangles, then all the shelves are "full", that is the width of each shelf is larger than $2 / 3$ (as all the width of any rectangle of $L_{H} \cup L_{X H}$ is lower than $1 / 3)$. Thus, we have $W\left(\pi_{0}^{i} \cap\left(L_{H} \cup L_{X H}\right)\right)>4 / 3>1$ (for $N_{1}+1 \leq i \leq N$ ). This implies that the total width of remaining rectangles of $L_{H} \cup L_{X H}$ (including those in strip $S_{N_{1}}$ ) is now lower than 1 . Thus, we can pack all of them in one shelf in $S_{N_{1}}$.

From now we assume that $H\left(L a y_{i}\right)>\frac{3 w}{2}$ for all $i<i_{1}$. This implies that $S\left(\pi_{0}^{i}\right)>v$ for $i<N_{1}$. Moreover, we have $2 N v \geq H\left(L_{W D}\right) \geq \sum_{i=1}^{N_{1}-1} H\left(\pi_{0}^{i} \cap L_{W D}\right)>\left(N_{1}-\right.$ 1) $2(3 v / 2)$, implying $N_{1}<\frac{2}{3} N+1$.

It remains now to pack $L_{H}^{1} \cup L_{X H}^{1}$. Notice that $\left(L_{H}^{1} \cup\right.$ $\left.L_{X H}^{1}\right) \cap L_{W D}=\emptyset$ (we say that $\left(L_{H}^{1} \cup L_{X H}^{1}\right)$ contains purely high and very high rectangles).

\subsection{Packing high and very high rectangles}

\subsubsection{Preliminaries}

Let $N_{2}=N-N_{1}$ denote the number of free strips after phase 1 . Roughly speaking, phase 2 packs shelves of high or very high rectangles in each of the $N_{2}$ last strips and merges some high or very high rectangles with the ones packed in strip $N_{1}$ (using the Merge procedure).

In this section we present a technique to fill $\gamma$ empty strips with high or very high rectangles. In the Section 3.5, we use this technique for $\gamma=N_{2}$ (using strips $S_{N_{1}+1}$ $\ldots S_{N}$ ) and an additional merging algorithm (that fills efficiently strip $S_{N_{1}}$ ) to pack $L_{H}^{1} \cup L_{X H}^{1}$.

Let us now introduce the procedure GreedyPack(X, seq). Given an ordered sequence of bins seq, GreedyPack creates for each empty bin $b \in s e q$ a shelf of rectangles of $X$ using CreateShel $f(X, 1)$ and packs it into $b$ (an example of a shelf packed in a bin is depicted Figure 3, Page 3). This procedure returns the last bin in which a shelf has been created, or null if no shelf is created. Notice that we will always use sequence of bins that have always width 1 , and the same height $h_{b}$ such that $\max _{r_{x} \in X} h_{x} \leq h_{b}$.

We now define the two sequences of bins $s e q_{X H}$ and $s e q_{H}$ that will be used by GreedyPack. Every bin of $s e q_{X H}$ (resp. $s e q_{H}$ ) will (possibly) contain one shelf of rectangles of $L_{X H}$ (resp. $\left.L_{H}\right)$. Notice that in a free strip it is possible to pack two bins of height $v$ (width of bins is always 1 ), three bins of height $2 v / 3$, or one bin of size $v$ and one bin of size $2 v / 3$. Thus, $s e q_{X H}$ is composed of $2 \gamma$ bins $\left(b_{1}, \ldots, b_{2 \gamma}\right)$ of height $v$, considering that we created two bins of height one in each of the strips $S_{1}, \ldots S_{\gamma}$. More precisely, for all $i$ we locate $b_{2 i-1}$ and $b_{2 i}$ in $S_{i}$, with $b_{2 i-x}$ at level $v(1-x)$ for $x \in\{0,1\}$. The sequence $\operatorname{seq}_{H}$ is composed of $3 \gamma$ bins $\left(b_{1}^{\prime}, \ldots, b_{3 \gamma}^{\prime}\right)$ of height $2 v / 3$, considering that we created three bins in each of the strips $S_{\gamma}, \ldots S_{1}$. It means that for all $i \geq 1$, bins $b_{3 i-2}^{\prime}, b_{3 i-1}^{\prime}$ and $b_{3 i}^{\prime}$ are located in $S_{\gamma-i+1}$, with $b_{3 i-x}^{\prime}$ at level $\frac{2 x v}{3}$ for $x \in[|0,2|]$. This sequences of bins will be used in Lemma 9, and later in phase 2 .

Finally, let us define the $\operatorname{Add}\left(X, S_{\text {ilast }_{\text {la }}}\right)$ procedure that packs the set of rectangles $X \subset L_{H} \backslash L_{W D}$ in $S_{i_{\text {last }}}$. As one can see in Lemma 9, $S_{i_{\text {last }}}$ is the last strip where Greedypack created a shelf. Thus, we assume for the moment that $S_{i_{\text {last }}}$ may only contain two different shapes of packing, and define the $A d d$ procedure accordingly. 
In the first case $S_{i_{\text {last }}}$ contains a first "full" shelf (full means that the surface of the shelf is at least $v / 2$ ) of rectangles of $L_{X H}$ at level 0 , and a shelf $s h$ of rectangles of $L_{X H}$ packed at level $v$, right justified. In this case, $A d d$ creates a shelf $s h_{1}$ using CreateShel $f(X, 1-W(s h))$ and preallocate $s h_{1}$ at level $v$, left justified.

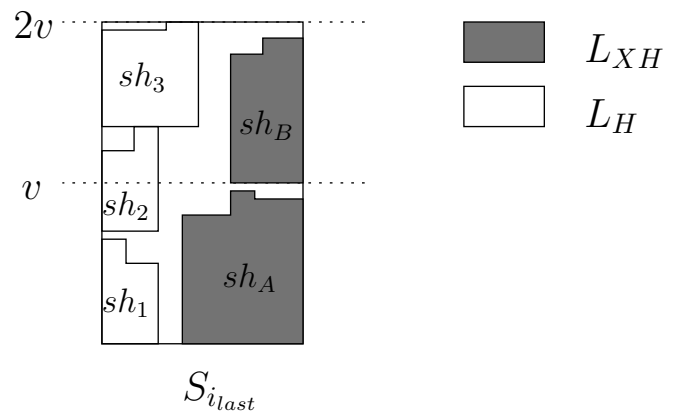

Figure 5. Example of the add procedure.

In the second case (see Figure 5), $S_{i_{\text {last }}}$ contains only a shelf of rectangles of $L_{X H}$ packed at level 0, right justified. In this case, $A d d$ first moves some rectangles from $s h$ to a new shelf $\widehat{s h}$ until $W(s h) \leq 2 / 3$. Then, $A d d$ packs (right justified) the widest of these two shelves (denoted by $s h_{A}$ ) at level 0 , and the other one (denoted by $s h_{B}$ ) at level $v$. Finally, $A d d$ creates two shelves $s h_{1}$ and $s h_{2}$ using CreateShelf $\left(X, 1-W\left(s h_{A}\right)\right)$ and one shelf $s h_{3}$ using CreateShelf $\left(X, 1-W\left(s h_{B}\right)\right)$. Then, $s h_{i}$ is packed at level $\frac{2 v(i-1)}{3}$, left justified. Notice that stacking shelves $s h_{1}, s h_{2}, s h_{3}$ does not exceed $2 v$.

We end these preliminaries with the following Lemma about the efficiency of $A d d$.

Lemma 8 Let $X \subset L_{H} \backslash L_{W D}$ and $S_{i}$ be a strip packed as expected for $\operatorname{Add}\left(X, S_{i}\right)$. Let $\pi_{0}^{i}$ denote the rectangles packed in $S_{i}$ before the call $A d d\left(X, S_{i}\right)$. If $X \neq \emptyset$ after calling the procedure, then $S\left(\pi_{0}^{i} \cup X\right)>v$.

Proof Remember that two cases are possible according to what is already packed in $S_{i}$ before the call. Let us first suppose that there is one full shelf (of area strictly larger than $v / 2$ ) of very high rectangles (at level 0 ) and another shelf $s h$ of very high rectangles at level $v$. Then, $X \neq \emptyset$ after the call implies that $W(X)>1-W(s h)$, and we have $S\left(\pi_{0}^{i} \cup X\right)>\frac{v}{2}+W(\operatorname{sh}) \frac{2 v}{3}+W(X) \frac{v}{2}>v$.

Let us now suppose that $S_{i}$ contains only one shelf $s h$ of $L_{X H}$ at level 0 . Let $s h_{A}, s h_{B}, s h_{1}, s h_{2}, s h_{3}$ be defined as described in the $A d d$ procedure. As $W\left(s h_{A}\right) \leq \frac{2}{3}$ $\left(W\left(s h_{b}\right) \leq \frac{2}{3}\right.$ is also true), and $X \cap L_{W D}=\emptyset, s h_{1}$ and $s h_{2}$ contain at least one rectangle, implying that $W\left(s h_{1}\right)$ and $W\left(s h_{2}\right)$ are strictly larger than $\frac{1-W\left(s h_{A}\right)}{2}$ according to Lemma 2. Moreover, $X \neq \emptyset$ after the call implies that after creating $s h_{1}$ and $s h_{2}$ the total width of remaining rectangles of $X$ was strictly larger than $1-W\left(s h_{B}\right)$. Putting this together, we get $S\left(\pi_{0}^{i} \cup X\right)>\left(1-W\left(s h_{A}\right)\right) \frac{v}{2}+(1-$ $\left.W\left(s h_{B}\right)\right) \frac{v}{2}+\left(W\left(s h_{A}\right)+W\left(s h_{B}\right)\right) \frac{2 v}{3}>v$.

\subsubsection{Filling $\gamma$ empty strips with high and very high rectangles}

The next lemma shows how to fill $\gamma$ free strips.

Lemma 9 Let $\widehat{L_{X H}} \subset L_{X H} \backslash L_{W D}$ and $\widehat{L_{H}} \subset L_{H} \backslash L_{W D}$ be two sets of rectangles that we have to pack. Suppose that we execute the following calls:

1. last $=$ GreedyPack $\left(\widehat{L_{X H}}, \operatorname{seq}_{X H}\right)$

2. GreedyPack $\left(\widehat{L_{H}}, \operatorname{seq}_{H}\right)$

3. $\operatorname{Add}\left(\widehat{L_{H}}, S_{i_{\text {last }}}\right)$ where $S_{i_{\text {last }}}$ denotes the strip containing the bin "last".

Then, we get the following properties:

- If $\widehat{L_{X H}} \neq \emptyset$ after 1 , then $S\left(\widehat{L_{X H}}\right)>\left(\gamma+\frac{1}{6}\right) v$

- Otherwise, if $\widehat{L_{H}} \neq \emptyset$ after 3 , then $S\left(\widehat{L_{X H}} \cup \widehat{L_{H}}\right)>$ $\gamma v$.

Remark 10 Let $X$ such that $X \cap L_{W D}=\emptyset$ and let sh denote a shelf created by CreateShel $f(X, 1)$, supposing that we did not run out of rectangle while creating the shelf. Then, according to Lemma 2, as at least three rectangles fit we have $W(s h)>3 / 4$. Moreover, if $X \subset L_{X H}$ then $S(s h)>v / 2$, and if $X \subset L_{H}$ then $S(s h)>3 v / 8$.

Proof [Proof of lemma 9] Let us first suppose that $\widehat{L_{X H}} \neq \emptyset$ after step 1 . It implies that after creating the first $2 \gamma-1$ shelves of width at least $3 / 4$ (according to Remark 10), the total remaining width of rectangles of $\widehat{L_{X H}}$ was strictly larger than 1 . Thus, $S\left(\widehat{\left.L_{X H}\right)}>\frac{3}{4}(2 \gamma-1) \frac{2 v}{3}+\frac{2 v}{3}=\right.$ $\left(\gamma+\frac{1}{6}\right) v$.

Let us now suppose that $\widehat{L_{X H}}=\emptyset$ and $\widehat{L_{H}} \neq \emptyset$ after step 3. Let $s h$ denote the shelf of rectangles of $\widehat{L_{X H}}$ contained in bin last. Remind that $i_{\text {last }}$ is the index of the strip containing last. For all $i \in\left[\left|1, i_{\text {last }}-1\right|\right]$, $S\left(\pi_{0}^{i} \cap\left(\widehat{L_{X H}} \cup \widehat{L_{H}}\right)\right)>2 \frac{v}{2}=v$. For all $i \in\left[\left|i_{\text {last }}+1, \gamma\right|\right]$, $S\left(\pi_{0}^{i} \cap\left(\widehat{L_{X H}} \cup \widehat{L_{H}}\right)\right)>3 \frac{3 v}{8}>v$. According to Lemma $8, \widehat{L_{H}} \neq \emptyset$ implies $S\left(\pi_{0}^{i_{\text {last }}} \cap \widehat{L_{H}}\right)>v$. Thus, we get that $S\left(\widehat{L_{X H}} \cup \widehat{L_{H}}\right)>\gamma v$. 


\subsection{Phase 2}

In phase 1 we preallocated $L_{W D}$ in strips $S_{1}, \ldots, S_{N_{1}}$. Recall that each layer created in phase 1 is sorted with the narrowest rectangles on the top. It remains now to preallocate $L_{X H}^{1} \cup L_{H}^{1}$ in $S_{N_{1}}, \ldots, S_{N}$.

Theorem 11 It is possible to preallocate $L_{X H}^{1} \cup L_{H}^{1}$ in $S_{N_{1}}, \ldots, S_{N}$ with a resulting height lower than $2 v$. Thus, our algorithm is a 2-approximation.

Proof Due to lack of space, we only sketch the proof of the theorem here and refer the reader to [4] for the complete case analysis.

An example of the final packing is depicted Figure 6. Phase 1 optimally filled the $\left(N_{1}-1\right)$ first strips using rectangles of $L_{W D}$. Phase 2 optimally filled strips $N_{1}+1$ to $i_{\text {last }}-1$ using two shelves of very high rectangles in each strip, and optimally filled strips $i_{\text {last }}+1$ to $N$ using three shelves of high rectangles in each strip. Strips $N_{1}$ and $i_{\text {last }}$ will be carefully filled according to a case by case analysis. According to Section 3.3, we know that the area packed in the $N_{1}-1$ first strips is greater than $\left(N_{1}-1\right) v$. The general idea is to pack $L_{X H}^{1} \cup L_{H}^{1}$ is to call GreedyPack (see Lemma 9) on strips $S_{N_{1}+1}, \ldots, S_{N}$. If $L_{X H}^{1}$ and $L_{H}^{1}$ are entirely packed by GreedyPack, then all the rectangles are packed in $2 v$. Otherwise, we can use Lemma 9 to claim that, $S\left(L_{X H}^{1} \cup L_{H}^{1}\right)>\left(N-N_{1}\right) v$ (or $\left.\left(N-N_{1}+\frac{1}{6}\right) v\right)$, and the remaining rectangles are packed by carefully studying what was packed in $S_{N_{1}}$ by phase 1 . Thus, we finish the proof using a case distinction according to the shape of the preallocation in $S_{N_{1}}$.

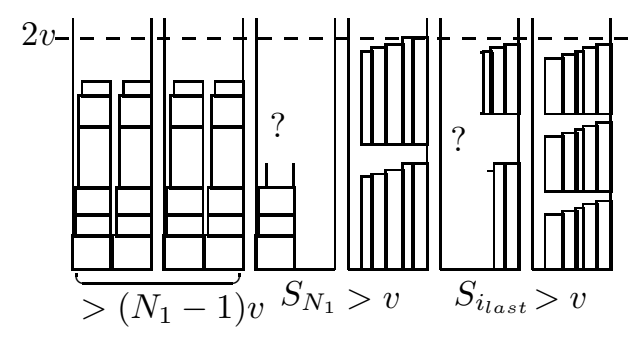

Figure 6. Example of a preallocation.

\subsection{Concluding remarks}

According to the main steps defined in Section 2.2, the previous 2 phases algorithm that preallocates $I^{\prime}$ is sufficient to get a 2-approximation. Indeed, we simply add rectangles of $I \backslash I^{\prime}$ using list algorithms defined in Section 2.2.

Let us now sketch the analysis of the running time of the preallocation algorithm. Phase 1 and Phase 2 run in
$\mathcal{O}(n \log (n)+N n)$ as for each strip creating a layer or a shelf can be done in $\mathcal{O}(n)$ (by sorting wide rectangles according to their heights before phase 1 , and sorting high rectangles according to their widths before phase 2). The list scheduling algorithms of Section 2.2.2 that turn $\pi_{0}$ into the final packing can be implemented in $\mathcal{O}(n \log (n))$ by using a global list (i.e. that refers to the $N$ strips) of currently "scheduled" rectangles, instead of scanning level by level and strip by strip (which is in $\mathcal{O}(N n \log (n)))$.

Finally, taking into account the repetitions due to the binary search on $v$ (where $\left.0 \leq v \leq n h_{\max }\right)$, the overall algorithm runs in $O\left(\log \left(n h_{\max }\right) n(N+\log (n))\right)$.

\section{References}

[1] N. Bansal, A. Caprara, K. Jansen, L. Prdel, and M. Sviridenko. How to maximize the total area of rectangle packed into a rectangle. In ISAAC, 2009.

[2] M. Bougeret, P.-F. Dutot, K. Jansen, C. Otte, and D. Trystram. Approximation algorithm for multiple strip packing. In Proceedings of the 7th Workshop on Approximation and Online Algorithms (WAOA), 2009.

[3] M. Bougeret, P.-F. Dutot, K. Jansen, C. Otte, and D. Trystram. Approximating the non-contiguous multiple organization packing problem. In Proceedings of the 6th IFIP International Conference on Theoretical Computer Science (TCS), 2010.

[4] M. Bougeret, P.-F. Dutot, K. Jansen, C. Robenek, and D. Trystram. Tight approximation for scheduling parallel jobs on identical clusters. LIRMM research report 12001 http: //hal-lirmm.ccsd.cnrs.fr/ lirmm-00656780.

[5] P.-F. Dutot and D. Trystram. Scheduling on hierarchical clusters using malleable tasks. In Proceedings of the thirteenth annual ACM symposium on Parallel algorithms and architectures, pages 199-208. ACM Press, 2001.

[6] D. Hochbaum and D. Shmoys. Using dual approximation algorithms for scheduling problems theoretical and practical results. Journal of the ACM (JACM), 34(1):144-162, 1987.

[7] D. Hochbaum and D. Shmoys. A polynomial approximation scheme for scheduling on uniform processors: Using the dual approximation approach. SIAM Journal on Computing, 17(3):539-551, 1988.

[8] K. Jansen. An EPTAS for scheduling jobs on uniform processors: using an MILP relaxation with a constant number of integral variables. Automata, Languages and Programming, pages 562-573, 2009.

[9] U. Schwiegelshohn, A. Tchernykh, and R. Yahyapour. Online scheduling in grids. In IEEE International Symposium on Parallel and Distributed Processing (IPDPS), pages 110, 2008.

[10] D. Ye, X. Han, and G. Zhang. On-Line Multiple-Strip Packing. In Proceedings of the 3rd International Conference on Combinatorial Optimization and Applications (COCOA), page 165. Springer, 2009.

[11] S. Zhuk. Approximate algorithms to pack rectangles into several strips. Discrete Mathematics and Applications, 16(1):73-85, 2006. 